\title{
The Generalized Gronwall Inequality and Its Application to Periodic Solutions of Integrodifferential Impulsive Periodic System on Banach Space
}

\author{
JinRong Wang, ${ }^{1}$ X. Xiang, ${ }^{1,2}$ W. Wei, ${ }^{2}$ and Qian Chen ${ }^{2}$ \\ ${ }^{1}$ College of Computer Science and Technology, Guizhou University, Guiyang, Guizhou 550025, China \\ ${ }^{2}$ College of Science, Guizhou University, Guiyang, Guizhou 550025, China \\ Correspondence should be addressed to JinRong Wang, wjr9668@126.com
}

Received 27 June 2008; Accepted 29 September 2008

Recommended by Ondřej Došlý

This paper deals with a class of integrodifferential impulsive periodic systems on Banach space. Using impulsive periodic evolution operator given by us, the $T_{0}$-periodic $P C$-mild solution is introduced and suitable Poincaré operator is constructed. Showing the compactness of Poincaré operator and using a new generalized Gronwall's inequality with impulse, mixed type integral operators and $B$-norm given by us, we utilize Leray-Schauder fixed point theorem to prove the existence of $T_{0}$-periodic $P C$-mild solutions. Our method is much different from methods of other papers. At last, an example is given for demonstration.

Copyright (C) 2008 JinRong Wang et al. This is an open access article distributed under the Creative Commons Attribution License, which permits unrestricted use, distribution, and reproduction in any medium, provided the original work is properly cited.

\section{Introduction}

It is well known that impulsive periodic motion is a very important and special phenomenon not only in natural science, but also in social science such as climate, food supplement, insecticide population, and sustainable development. Periodic system with applications on finite-dimensional spaces has been extensively studied. Particularly, impulsive periodic systems on finite-dimensional spaces are considered and some important results (such as the existence and stability of periodic solution, the relationship between bounded solution and periodic solution, and robustness by perturbation) are obtained (see [1-4]).

Since the end of last century, many researchers pay great attention to impulsive systems on infinite-dimensional spaces. Particulary, Ahmed et al. investigated optimal control problems of system governed by impulsive system (see [5-8]). Many authors including us also gave a series of results for semilinear (integrodifferential, strongly nonlinear) impulsive systems and optimal control problems (see [9-20]). 
Although, there are some papers on periodic solution for periodic system on infinitedimensional spaces (see $[12,21-23]$ ) and some results discussing integrodifferential system on finite Banach space and infinite Banach space (see [11, 13]). To our knowledge, integrodifferential impulsive periodic systems on infinite-dimensional spaces (with unbounded operator) have not been extensively investigated. Recently, we discuss the impulsive periodic system and integrodifferential impulsive system on infinite-dimensional spaces. Linear impulsive evolution operator is constructed and $T_{0}$-periodic $P C$-mild solution is introduced. The existence of periodic solutions, alternative theorem (criteria of Massera type), asymptotical stability, and robustness by perturbation is established (see [24-26]). For semilinear impulsive periodic system, a suitable Poincaré operator is constructed which verifies its compactness and continuity. By virtue of a generalized Gronwall inequality with mixed integral operator and impulse given by us, the estimate of the $P C$-mild solutions is derived. Some fixed point theorems such as Banach fixed point theorem and Horn fixed point theorem are applied to obtain the existence of periodic $P C$-mild solutions, respectively (see $[27,28])$. For integrodifferential impulsive system, the existence of $P C$-mild solutions and optimal controls is presented (see [15]).

Herein, we go on studying the following integrodifferential impulsive periodic system

$$
\begin{gathered}
\dot{x}(t)=A x(t)+f\left(t, x, \int_{0}^{t} g(t, s, x) d s\right), \quad t \neq \tau_{k}, \\
\Delta x(t)=B_{k} x(t)+c_{k}, \quad t=\tau_{k} .
\end{gathered}
$$

on infinite-dimensional Banach space $X$, where $0=\tau_{0}<\tau_{1}<\tau_{2}<\cdots<\tau_{k} \cdots ; \lim _{k \rightarrow \infty} \tau_{k}=\infty$, $\tau_{k+\delta}=\tau_{k}+T_{0} ; \Delta x\left(\tau_{k}\right)=x\left(\tau_{k}^{+}\right)-x\left(\tau_{k}^{-}\right), k \in \mathbb{Z}_{0}^{+} ; T_{0}$ is a fixed positive number; and $\delta \in \mathbb{N}$ denoted the number of impulsive points between 0 and $T_{0}$. The operator $A$ is the infinitesimal generator of a $C_{0}$-semigroup $\{T(t), t \geq 0\}$ on $X ; f$ is a $T_{0}$-periodic, with respect to $t \in[0+$ $\infty)$, Carathédory function; $g$ is a continuous function from $[0, \infty) \times[0, \infty) \times X$ to $X$ and is $T_{0}$-periodic in $t$ and $s$; and $B_{k+\delta}=B_{k}, c_{k+\delta}=c_{k}$. This paper is mainly concerned with the existence of periodic solutions for integrodifferential impulsive periodic system on infinitedimensional Banach space $X$.

In this paper, we use Leray-Schauder fixed point theorem to obtain the existence of periodic solutions for integrodifferential impulsive periodic system (1.1). First, by virtue of impulsive evolution operator corresponding to linear homogeneous impulsive system, we construct a new Poincaré operator $P$ for integrodifferential impulsive periodic system (1.1), then we overcome some difficulties to show the compactness of Poincaré operator $P$ which is very important. By a new generalized Gronwall inequality with impulse, mixedtype integral operators, and $B$-norm given by us, the estimate of fixed point set $\{x=\lambda P x, \lambda \in$ $[0,1]\}$ is established. Therefore, the existence of $T_{0}$-periodic $P C$-mild solutions for impulsive integrodifferential periodic system is shown.

In order to obtain the existence of periodic solutions, many authors use Horn fixed point theorem or Banach fixed point theorem. However, the conditions for Horn fixed point theorem are not easy to be verified sometimes and the conditions for Banach fixed point theorem are too strong. Our method is much different from others', and we give a new way to show the existence of periodic solutions. In addition, the new generalized Gronwall inequality with impulse, mixed-type integral operator, and B-norm given by us, which can be used in other problems, have played an essential role in the study of nonlinear problems on infinite-dimensional spaces. 
This paper is organized as follows. In Section 2, some results of linear impulsive periodic system and properties of impulsive periodic evolution operator corresponding to homogeneous linear impulsive periodic system are recalled. In Section 3, the new generalized Gronwall inequality with impulse, mixed-type integral operator, and $B$-norm are established. In Section 4, the $T_{0}$-periodic $P C$-mild solution for integrodifferential impulsive periodic system (1.1) is introduced. We construct the suitable Poincaré operator $P$ and give the relation between $T_{0}$-periodic $P C$-mild solution and the fixed point of $P$. After showing the compactness of the Poincaré operator $P$ and obtaining the boundedness of the fixed point set $\{x=\lambda P x, \lambda \in[0,1]\}$ by virtue of the generalized Gronwall inequality, we can use LeraySchauder fixed point theorem to establish the existence of $T_{0}$-periodic $P C$-mild solutions for integrodifferential impulsive periodic system. At last, an example is given to demonstrate the applicability of our result.

\section{Linear impulsive periodic system}

In order to study the integrodifferential impulse periodic system, we first recall some results about linear impulse periodic system here. Let $X$ be a Banach space. $£(X)$ denotes the space of linear operators in $X ; £_{b}(X)$ denotes the space of bounded linear operators in $X . £_{b}(X)$ is the Banach space with the usual supremum norm. Define $\widetilde{D}=\left\{\tau_{1}, \ldots, \tau_{\delta}\right\} \subset\left[0, T_{0}\right]$, where $\delta \in \mathbb{N}$ denotes the number of impulsive points between $\left[0, T_{0}\right]$. We introduce $P C\left(\left[0, T_{0}\right] ; X\right) \equiv\{x$ : $\left[0, T_{0}\right] \rightarrow X \mid x$ to be continuous at $t \in\left[0, T_{0}\right] \backslash \widetilde{D} ; x$ is continuous from left and has right-hand limits at $t \in \tilde{D}\}$; and $P C^{1}\left(\left[0, T_{0}\right] ; X\right) \equiv\left\{x \in P C\left(\left[0, T_{0}\right] ; X\right) \mid \dot{x} \in P C\left(\left[0, T_{0}\right] ; X\right)\right\}$. Set

$$
\|x\|_{P C}=\max \left\{\sup _{t \in\left[0, T_{0}\right]}\|x(t+0)\|, \sup _{t \in\left[0, T_{0}\right]}\|x(t-0)\|\right\}, \quad\|x\|_{P C^{1}}=\|x\|_{P C}+\|\dot{x}\|_{P C} .
$$

It can be seen that endowed with the norm $\|\cdot\|_{P C}\left(\|\cdot\|_{P C^{1}}\right), P C\left(\left[0, T_{0}\right] ; X\right)\left(P C^{1}\left(\left[0, T_{0}\right] ; X\right)\right)$ is a Banach space.

Firstly, we consider homogeneous linear impulsive periodic system

$$
\begin{aligned}
\dot{x}(t) & =A x(t), \quad t \neq \tau_{k}, \\
\Delta x(t) & =B_{k} x(t), \quad t=\tau_{k} .
\end{aligned}
$$

We introduce the following assumption [H1].

[H1.1] $A$ is the infinitesimal generator of a $C_{0}$-semigroup $\{T(t), t \geq 0\}$ on $X$ with domain $D(A)$.

[H1.2] There exists $\delta$ such that $\tau_{k+\delta}=\tau_{k}+T_{0}$.

[H1.3] For each $k \in \mathbb{Z}_{0}^{+}, B_{k} \in £_{b}(X)$ and $B_{k+\delta}=B_{k}$.

In order to study system (2.2), we need to consider the associated Cauchy problem

$$
\begin{gathered}
\dot{x}(t)=A x(t), \quad t \in\left[0, T_{0}\right] \backslash \tilde{D}, \\
\Delta x\left(\tau_{k}\right)=B_{k} x\left(\tau_{k}\right), \quad k=1,2, \ldots, \delta, \\
x(0)=\bar{x} .
\end{gathered}
$$


If $\bar{x} \in D(A)$ and $D(A)$ is an invariant subspace of $B_{k}$, using Theorem 5.2.2, (see [29, page 144]), step by step, one can verify that the Cauchy problem (2.3) has a unique classical solution $x \in P C^{1}\left(\left[0, T_{0}\right] ; X\right)$ represented by $x(t)=S(t, 0) \bar{x}$, where

$$
S(\cdot, \cdot): \Delta=\left\{(t, \theta) \in\left[0, T_{0}\right] \times\left[0, T_{0}\right] \mid 0 \leq \theta \leq t \leq T_{0}\right\} \longrightarrow £_{b}(X)
$$

given by

$S(t, \theta)=\left\{\begin{array}{lr}T(t-\theta), & \tau_{k-1} \leq \theta \leq t \leq \tau_{k}, \\ T\left(t-\tau_{k}^{+}\right)\left(I+B_{k}\right) T\left(\tau_{k}-\theta\right), & \tau_{k-1} \leq \theta<\tau_{k}<t \leq \tau_{k+1}, \\ T\left(t-\tau_{k}^{+}\right)\left[\prod_{\theta<\tau_{j}<t}\left(I+B_{j}\right) T\left(\tau_{j}-\tau_{j-1}^{+}\right)\right]\left(I+B_{i}\right) T\left(\tau_{i}-\theta\right), & \\ \tau_{i-1} \leq \theta<\tau_{i} \leq \cdots<\tau_{k}<t \leq \tau_{k+1} .\end{array}\right.$

The operator $\{S(t, \theta),(t, \theta) \in \Delta\}$ is called impulsive evolution operator associated with $\left\{B_{k} ; \tau_{k}\right\}_{k=1}^{\infty}$.

Now we introduce the PC-mild solution of Cauchy problem (2.3) and $T_{0}$-periodic $P C$ mild solution of the system (2.2).

Definition 2.1. For every $\bar{x} \in X$, the function $x \in P C\left(\left[0, T_{0}\right] ; X\right)$ given by $x(t)=S(t, 0) \bar{x}$ is said to be the $P C$-mild solution of the Cauchy problem (2.3).

Definition 2.2. A function $x \in P C([0,+\infty) ; X)$ is said to be a $T_{0}$-periodic $P C$-mild solution of system (2.2) if it is a PC-mild solution of Cauchy problem (2.3) corresponding to some $\bar{x}$ and $x\left(t+T_{0}\right)=x(t)$ for $t \geq 0$.

The following lemma gives the properties of the impulsive evolution operator $\{S(t, \theta),(t, \theta) \in \Delta\}$ associated with $\left\{B_{k} ; \tau_{k}\right\}_{k=1}^{\infty}$ which are widely used in sequel.

Lemma 2.3 (see [24, Lemma 1]). Impulsive evolution operator $\{S(t, \theta),(t, \theta) \in \Delta\}$ has the following properties.

(1) For $0 \leq \theta \leq t \leq T_{0}, S(t, \theta) \in £_{b}(X)$, that is, $\sup _{0 \leq \theta \leq t \leq T_{0}}\|S(t, \theta)\| \leq M_{T_{0}}$, where $M_{T_{0}}>0$.

(2) For $0 \leq \theta<r<t \leq T_{0}, r \neq \tau_{k}, S(t, \theta)=S(t, r) S(r, \theta)$.

(3) For $0 \leq \theta \leq t \leq T_{0}$ and $N \in Z_{0}^{+}, S\left(t+N T_{0}, \theta+N T_{0}\right)=S(t, \theta)$.

(4) For $0 \leq t \leq T_{0}$ and $N \in Z_{0}^{+}, S\left(N T_{0}+t, 0\right)=S(t, 0)\left[S\left(T_{0}, 0\right)\right]^{N}$.

(5) If $\{T(t), t \geq 0\}$ is a compact semigroup in $X$, then $S(t, \theta)$ is a compact operator for $0 \leq \theta<$ $t \leq T_{0}$.

Here, we note that system (2.2) has a $T_{0}$-periodic $P C$-mild solution $x$ if and only if $S\left(T_{0}, 0\right)$ has a fixed point. The impulsive evolution operator $\{S(t, \theta),(t, \theta) \in \Delta\}$ can be used to reduce the existence of $T_{0}$-periodic $P C$-mild solutions for linear impulsive periodic system to the existence of fixed points for an operator equation. This implies that we can build up 
the new framework to study the periodic $P C$-mild solutions for integrodifferential impulsive periodic system on Banach space.

Consider nonhomogeneous linear impulsive periodic system

$$
\begin{array}{ll}
\dot{x}(t)=A x(t)+f(t), & t \neq \tau_{k}, \\
\Delta x(t)=B_{k} x(t)+c_{k}, & t=\tau_{k},
\end{array}
$$

and the associated Cauchy problem

$$
\begin{gathered}
\dot{x}(t)=A x(t)+f(t), \quad t \in\left[0, T_{0}\right] \backslash \tilde{D}, \\
\Delta x\left(\tau_{k}\right)=B_{k} x\left(\tau_{k}\right)+c_{k}, \quad k=1,2, \ldots, \delta, \\
x(0)=\bar{x} .
\end{gathered}
$$

where $f \in L^{1}\left(\left[0, T_{0}\right] ; X\right), f\left(t+T_{0}\right)=f(t)$ and $c_{k+\delta}=c_{k}$.

Now we introduce the $P C$-mild solution of Cauchy problem (2.7) and $T_{0}$-periodic $P C$ mild solution of system (2.6).

Definition 2.4. A function $x \in P C\left(\left[0, T_{0}\right] ; X\right)$, for finite interval $\left[0, T_{0}\right]$, is said to be a $P C$-mild solution of the Cauchy problem (2.6) corresponding to the initial value $\bar{x} \in X$ and input $f \in L^{1}\left(\left[0, T_{0}\right] ; X\right)$ if $x$ is given by

$$
x(t)=S(t, 0) \bar{x}+\int_{0}^{t} S(t, \theta) f(\theta) d \theta+\sum_{0 \leq \tau_{k}<t} S\left(t, \tau_{k}^{+}\right) c_{k} .
$$

Definition 2.5. A function $x \in P C([0,+\infty) ; X)$ is said to be a $T_{0}$-periodic $P C$-mild solution of system (2.6) if it is a PC-mild solution of Cauchy problem (2.7) corresponding to some $\bar{x}$ and $x\left(t+T_{0}\right)=x(t)$ for $t \geq 0$.

\section{The generalized Gronwall inequality}

In order to use Leray-Schauder theorem to show the existence of periodic solutions, we need a new generalized Gronwall inequality with impulse, mixed-type integral operator, and $B$ norm which is much different from classical Gronwall inequality and can be used in other problems (such as discussion on integrodifferential equation of mixed type, see [15]). It will play an essential role in the study of nonlinear problems on infinite-dimensional spaces.

We first introduce the following generalized Gronwall inequality with impulse and $B$-norm.

Lemma 3.1. Let $x \in P C([0, \infty), X)$ and satisfy the following inequality:

$$
\|x(t)\| \leq a+b \int_{0}^{t}\|x(\theta)\|^{\lambda_{1}} d \theta+d \int_{0}^{t}\left\|x_{\theta}\right\|_{B}^{\lambda_{3}} d \theta
$$


where $a, b, d \geq 0,0 \leq \lambda_{1}, \lambda_{3} \leq 1$ are constants, and $\left\|x_{\theta}\right\|_{B}=\sup _{0 \leq \xi \leq \theta}\|x(\xi)\|$. Then

$$
\|x(t)\| \leq(a+1) e^{(b+c) t}
$$

Proof. (i) For $0 \leq \lambda_{1}, \lambda_{3}<1$, let $\lambda=\max \left\{\lambda_{1}, \lambda_{3}\right\} \in[0,1)$ and

$$
y(t)= \begin{cases}1, & \|x(t)\| \leq 1 \\ x(t), & \|x(t)\|>1\end{cases}
$$

Then

$$
\|x(t)\| \leq\|y(t)\| \leq(a+1)+b \int_{0}^{t}\|y(\theta)\|^{\lambda} d \theta+d \int_{0}^{t}\left\|y_{\theta}\right\|_{B}^{\lambda} d \theta \quad \forall t \in\left[0, T_{0}\right] .
$$

Using (3.4), we obtain

$$
\left\|y_{t}\right\|_{B}^{\curlywedge} \leq(a+1)+(b+d) \int_{0}^{t}\left\|y_{\theta}\right\|_{B}^{\curlywedge} d \theta .
$$

\section{Define}

$$
u(t)=(a+1)+(b+d) \int_{0}^{t}\left\|y_{\theta}\right\|_{B}^{\lambda} d \theta
$$

we get

$$
\begin{gathered}
\dot{u}(t)=(b+d)\left\|y_{t}\right\|_{B^{\prime}}^{\lambda} \quad t \neq \tau_{k}, \\
u(0)=a+1, \quad u\left(\tau_{k}+0\right)=u\left(\tau_{k}\right) .
\end{gathered}
$$

Since $\left\|y_{t}\right\|_{B}^{\lambda} \leq u(t)$, we then have

$$
\begin{gathered}
\dot{u}(t) \leq(b+d) u(t), \quad t \neq \tau_{k}, \\
u(0)=a+1, \quad u\left(\tau_{k}+0\right)=u\left(\tau_{k}\right) .
\end{gathered}
$$

For $t \in\left(\tau_{k}, \tau_{k+1}\right]$, by (3.8), we obtain

$$
u(t) \leq u\left(\tau_{k}+0\right) e^{(b+d)\left(t-\tau_{k}\right)}=u\left(\tau_{k}\right) e^{(b+d)\left(t-\tau_{k}\right)},
$$


further,

$$
u(t) \leq(a+1) e^{(b+d) t}
$$

thus,

$$
\|x(t)\| \leq\left\|y_{t}\right\|_{B} \leq(a+1) e^{(b+d) t}
$$

(ii) For $\lambda_{1}=\lambda_{3}=1$, we only need to define

$$
u_{1}(t)=a+(b+d) \int_{0}^{t}\left\|x_{\theta}\right\|_{B} d \theta
$$

Similar to the proof in (i), one can obtain

$$
\|x(t)\| \leq\left\|x_{t}\right\|_{B} \leq a e^{(b+d) t}
$$

Combining (i) and (ii), one can complete the proof.

Using Gronwall's inequality with impulse and B-norm, we can obtain the following new generalized Gronwall Lemma.

Lemma 3.2. Let $x \in P C\left(\left[0, T_{0}\right] ; X\right)$ satisfy the following inequality:

$$
\begin{aligned}
& \|x(t)\| \\
& \quad \leq a+b \int_{0}^{t}\|x(\theta)\|^{\lambda_{1}} d \theta+c \int_{0}^{T_{0}}\|x(\theta)\|^{\lambda_{2}} d \theta+d \int_{0}^{t}\left\|x_{\theta}\right\|_{B}^{\lambda_{3}} d \theta+e \int_{0}^{T_{0}}\left\|x_{\theta}\right\|_{B}^{\lambda_{4}} d \theta \quad \forall t \in\left[0, T_{0}\right],
\end{aligned}
$$

where $\lambda_{1}, \lambda_{3} \in[0,1], \lambda_{2}, \lambda_{4} \in[0,1), a, b, c, d, e \geq 0$ are constants. Then there exists a constant $M^{*}>0$ such that

$$
\|x(t)\| \leq M^{*}
$$

Proof. By Lemma 3.1, we obtain that

$$
\|x(t)\| \leq\|y(t)\| \leq\left\|y_{t}\right\|_{B} \leq e^{(b+d) t}\left((a+1)+c \int_{0}^{T_{0}}\|y(\theta)\|^{\lambda} d \theta+e \int_{0}^{T_{0}}\left\|y_{\theta}\right\|_{B}^{\lambda} d \theta\right),
$$


where

$$
\begin{gathered}
y(t)= \begin{cases}1, & \|x(t)\| \leq 1, \\
x(t), & \|x(t)\|>1,\end{cases} \\
\lambda= \begin{cases}\max \left\{\lambda_{1}, \lambda_{2}, \lambda_{3}, \lambda_{4}\right\} \in[0,1), & \text { if } \lambda_{1}, \lambda_{2}, \lambda_{3}, \lambda_{4} \in[0,1), \\
\max \left\{\lambda_{2}, \lambda_{4}\right\} \in[0,1), & \text { if } \lambda_{1}=\lambda_{3}=1, \lambda_{2}, \lambda_{4} \in[0,1) .\end{cases}
\end{gathered}
$$

Define

$q(t)$

$\equiv e^{(b+d) T_{0}}\left((a+1)+c \int_{0}^{t}\|y(\theta)\|^{\lambda} d \theta+c \int_{0}^{T_{0}}\|y(\theta)\|^{\lambda} d \theta+e \int_{0}^{t}\left\|y_{\theta}\right\|_{B}^{\lambda} d \theta+e \int_{0}^{T_{0}}\left\|y_{\theta}\right\|_{B}^{\lambda} d \theta\right)$,

then $q$ is a monotone increasing function and

$$
\begin{aligned}
& \dot{q}(t) \\
& \quad=e^{(b+d) T_{0}}\left(c\|y(t)\|^{\lambda}+e\left\|y_{t}\right\|_{B}^{\lambda}\right) \leq(c+e) e^{(b+d) T_{0}}\left(\|y(t)\|^{\lambda}+\left\|y_{t}\right\|_{B}^{\lambda}\right) \leq 2(c+e) e^{(b+d) T_{0}} q^{\lambda}(t) .
\end{aligned}
$$

Consider

$$
\frac{d}{d t} q^{1-\lambda}(t)=(1-\lambda) q^{-\lambda}(t) \dot{q}(t) \leq 2(c+e) e^{(b+d) T_{0}}(1-\lambda) .
$$

Integrating from 0 to $t$, we obtain

$$
q^{1-\lambda}(t)-q^{1-\lambda}(0) \leq 2(c+e) e^{(b+d) T_{0}}(1-\lambda) t
$$

that is,

$$
q(t) \leq\left[q^{1-\lambda}(0)+2(c+e) e^{(b+d) T_{0}}(1-\lambda) t\right]^{1 /(1-\lambda)} .
$$

On the other hand,

$$
\begin{aligned}
& 2 q(0)=2 e^{(b+d) T_{0}}\left((a+1)+c \int_{0}^{T_{0}}\|y(\theta)\|^{\lambda} d \theta+e \int_{0}^{T_{0}}\left\|y_{\theta}\right\|_{B}^{\lambda} d \theta\right) \\
& q\left(T_{0}\right)=e^{(b+d) T_{0}}\left((a+1)+2 c \int_{0}^{T_{0}}\|y(\theta)\|^{\lambda} d \theta+2 e \int_{0}^{T_{0}}\left\|y_{\theta}\right\|_{B}^{\lambda} d \theta\right) .
\end{aligned}
$$


JinRong Wang et al.

Now, we observe that

$$
2 q(0)-e^{(b+d) T_{0}}(a+1)=q\left(T_{0}\right) \leq\left[q^{1-\lambda}(0)+2(c+e) e^{(b+d) T_{0}} T_{0}(1-\lambda)\right]^{1 /(1-\lambda)} .
$$

As a result, we get

$$
\left(2 q(0)-e^{(b+d) T_{0}}(a+1)\right)^{1-\lambda}-q^{1-\lambda}(0) \leq 2(c+e) e^{(b+d) T_{0}} T_{0}(1-\lambda) .
$$

Letting

$$
\Upsilon(z)=\left(2 z-e^{(b+d) T_{0}}(a+1)\right)^{1-\lambda}-z^{1-\lambda}-2(c+e) e^{(b+d) T_{0}} T_{0}(1-\lambda),
$$

we have $\Upsilon \in C\left(\left[e^{(b+d) T_{0}}(a+1) / 2,+\infty\right) ; \mathbb{R}\right)$ and $\Upsilon\left(e^{(b+d) T_{0}}(a+1) / 2\right)<0$. Moreover,

$$
\lim _{z \rightarrow+\infty} \frac{\Upsilon(z)}{z^{1-\lambda}}=2^{1-\lambda}-1>0
$$

Hence, there exists enough large $z_{0}>e^{(b+d) T_{0}}(a+1) / 2>0$ such that $\Upsilon(z)>0$ for arbitrary $z \geq z_{0}$. Meanwhile, $\Upsilon(q(0)) \leq 0$. Thus, $q(0) \leq z_{0}$.

As a result, we obtain

$$
\begin{aligned}
\|x(t)\| & \leq\|y(t)\| \leq q\left(T_{0}\right)=2 q(0)-e^{(b+d) T_{0}}(a+1) \\
& \leq 2 z_{0}-e^{(b+d) T_{0}}(a+1) \equiv M^{*}>0 \quad \forall t \in\left[0, T_{0}\right] .
\end{aligned}
$$

\section{Periodic solutions of integrodifferential impulsive periodic system}

In this section, we consider the following integrodifferential impulsive periodic system:

$$
\begin{gathered}
\dot{x}(t)=A x(t)+f\left(t, x, \int_{0}^{t} g(t, s, x) d s\right), \quad t \neq \tau_{k}, \\
\Delta x(t)=B_{k} x(t)+c_{k}, \quad t=\tau_{k} .
\end{gathered}
$$

and the associated Cauchy problem

$$
\begin{gathered}
\dot{x}(t)=A x(t)+f\left(t, x, \int_{0}^{t} g(t, s, x) d s\right), \quad t \in\left[0, T_{0}\right] \backslash \tilde{D}, \\
\Delta x\left(\tau_{k}\right)=B_{k} x\left(\tau_{k}\right)+c_{k}, \quad k=1,2, \ldots, \delta, \\
x(0)=\bar{x} .
\end{gathered}
$$


By virtue of the expression of the $P C$-mild solution of the Cauchy problem (2.7), we can introduce the $P C$-mild solution of the Cauchy problem (4.2).

Definition 4.1. A function $x \in P C\left(\left[0, T_{0}\right] ; X\right)$ is said to be a $P C$-mild solution of the Cauchy problem (4.2) corresponding to the initial value $\bar{x} \in X$ if $x$ satisfies the following integral equation:

$$
\begin{aligned}
x(t)= & S(t, 0) \bar{x}+\int_{0}^{t} S(t, \theta) f\left(\theta, x(\theta), \int_{0}^{\theta} g(\theta, s, x(s)) d s\right) d \theta \\
& +\sum_{0 \leq \tau_{k}<t} S\left(t, \tau_{k}^{+}\right) c_{k} \quad \text { for } t \in\left[0, T_{0}\right] .
\end{aligned}
$$

Now, we introduce the $T_{0}$-periodic $P C$-mild solution of system (4.1).

Definition 4.2. A function $x \in P C([0,+\infty) ; X)$ is said to be a $T_{0}$-periodic $P C$-mild solution of system (4.1) if it is a PC-mild solution of Cauchy problem (4.2) corresponding to some $\bar{x}$ and $x\left(t+T_{0}\right)=x(t)$ for $t \geq 0$.

Assumption [H2] includes the following.

[H2.1] $f:[0,+\infty) \times X \times X \rightarrow X$ satisfies the following.

(i) For each $(x, y) \in X \times X, t \rightarrow f(t, x, y)$ is measurable.

(ii) For each $\rho>0$, there exists $L_{f}(\rho)>0$ such that, for almost all $t \in[0,+\infty)$ and all $x_{1}$, $x_{2}, y_{1}, y_{2} \in X,\left\|x_{1}\right\|,\left\|x_{2}\right\|,\left\|y_{1}\right\|,\left\|y_{2}\right\| \leq \rho$, we have

$$
\left\|f\left(t, x_{1}, y_{1}\right)-f\left(t, x_{2}, y_{2}\right)\right\| \leq L_{f}(\rho)\left(\left\|x_{1}-x_{2}\right\|+\left\|y_{1}-y_{2}\right\|\right)
$$

[H2.2] There exists a positive constant $M_{f}$ such that

$$
\|f(t, x, y)\| \leq M_{f}(1+\|x\|+\|y\|) \quad \forall x, y \in X .
$$

[H2.3] $f(t, x, y)$ is $T_{0}$-periodic in $t$, that is, $f\left(t+T_{0}, x, y\right)=f(t, x, y), t \geq 0$.

[H2.4] Let $D=\{(t, s) \in[0+\infty) \times[0+\infty) ; 0 \leq s \leq t\}$. The function $g: D \times X \rightarrow X$ is continuous for each $\rho>0$, there exists $L_{g}(\rho)>0$ such that, for each $(t, s) \in D$ and each $x, y \in X$ with $\|x\|,\|y\| \leq \rho$, we have

$$
\|g(t, s, x)-g(t, s, y)\| \leq L_{g}(\rho)\|x-y\| .
$$

[H2.5] There exists a positive constant $M_{g}$ such that

$$
\|g(t, s, x)\| \leq M_{g}(1+\|x\|) \quad \forall x, y \in X .
$$



and

[H2.6] $g(t, s, x)$ are $T_{0}$-periodic in $t$ and $s$, that is, $g\left(t+T_{0}, s+T_{0}, x\right)=g(t, s, x), t \geq s \geq 0$

$$
\int_{0}^{T_{0}} g(t, s, x) d s=0, \quad t \geq s \geq 0
$$

[H2.7] For each $k \in \mathbb{Z}_{0}^{+}$and $c_{k} \in X$, there exists $\delta \in \mathbb{N}$ such that $c_{k+\delta}=c_{k}$.

Lemma 4.3. Under assumptions [H2.4] and [H2.5], one has the following properties:

(1) $\int_{0} g(\cdot, s, x(s)) d s: P C\left(\left[0, T_{0}\right] ; X\right) \rightarrow P C\left(\left[0, T_{0}\right] ; X\right)$.

(2) For all $x_{1}, x_{2} \in P C\left(\left[0, T_{0}\right] ; X\right)$ and $\left\|x_{1}\right\|_{P C\left(\left[0, T_{0}\right] ; X\right)},\left\|x_{2}\right\|_{P C\left(\left[0, T_{0}\right] ; X\right)} \leq \rho$,

$$
\left\|\int_{0}^{t} g\left(t, s, x_{1}(s)\right) d s-\int_{0}^{t} g\left(t, s, x_{2}(s)\right) d s\right\| \leq L_{g}(\rho) T_{0}\left\|x_{1}-x_{2}\right\|_{B} .
$$

(3) For $x \in P C\left(\left[0, T_{0}\right] ; X\right)$,

$$
\left\|\int_{0}^{t} g(t, s, x(s)) d s\right\| \leq M_{g} T_{0}\left(1+\left\|x_{t}\right\|_{B}\right) .
$$

Proof. (1) Since $g$ is continuous in its variables and satisfies linear growth conditions, one can verify that $\int_{0} g(\cdot, s, x(s)) d s$ maps $P C\left(\left[0, T_{0}\right] ; X\right)$ to $P C\left(\left[0, T_{0}\right] ; X\right)$.

(2) Let $x_{1}, x_{2} \in P C\left(\left[0, T_{0}\right] ; X\right),\left\|x_{1}\right\|_{P C\left(\left[0, T_{0}\right] ; X\right)},\left\|x_{2}\right\|_{P C\left(\left[0, T_{0}\right] ; X\right)} \leq \rho$, we have

$$
\begin{aligned}
\left\|\int_{0}^{t} g\left(t, s, x_{1}(s)\right) d s-\int_{0}^{t} g\left(t, s, x_{2}(s)\right) d s\right\| & \leq \int_{0}^{t}\left\|g\left(t, s, x_{1}(s)\right)-g\left(t, s, x_{2}(s)\right)\right\| d s \\
& \leq \int_{0}^{t} L_{g}(\rho)\left\|x_{1}(s)-x_{2}(s)\right\| d s \\
& \leq L_{g}(\rho) t\left\|\left(x_{1}\right)_{t}-\left(x_{2}\right)_{t}\right\|_{B} \\
& \leq L_{g}(\rho) T_{0}\left\|\left(x_{1}\right)_{t}-\left(x_{2}\right)_{t}\right\|_{B} .
\end{aligned}
$$

(3) For $x \in P C\left(\left[0, T_{0}\right] ; X\right)$,

$$
\begin{aligned}
\left\|\int_{0}^{t} g(t, s, x(s)) d s\right\| & \leq M_{g} \int_{0}^{t}(1+\|x(s)\|) d s \\
& \leq M_{g} t\left(1+\left\|x_{t}\right\|_{B}\right) \\
& \leq M_{g} T_{0}\left(1+\left\|x_{t}\right\|_{B}\right) .
\end{aligned}
$$

Now we present the existence of PC-mild solution for system (4.2). 
Theorem 4.4. Assumptions [H1.1], [H2.1], [H2.4], and [H2.5] hold. Then system (4.2) has a unique $P C$-mild solution given by

$$
x(t, \bar{x})=S(t, 0) \bar{x}+\int_{0}^{t} S(t, \theta) f\left(\theta, x(\theta), \int_{0}^{\theta} g(\theta, s, x(s)) d s\right) d \theta+\sum_{0 \leq \tau_{k}<t} S\left(t, \tau_{k}^{+}\right) c_{k} .
$$

Proof. A similar result is given by Wei et al. [15]. Thus, we only sketch the proof here. In order to make the process clear, we divide it into three steps.

Step 1. We consider the following general integrodifferential equation without impulse

$$
\begin{gathered}
\dot{x}(t)=A x(t)+f\left(t, x, \int_{0}^{t} g(t, s, x) d s\right), \quad t \in[s, \tau], \\
x(s)=\bar{x} \in X .
\end{gathered}
$$

In order to obtain the local existence of mild solution for system (4.14), we only need to set up the framework for use of the contraction mapping theorem. Consider the ball given by

$$
\mathfrak{B}=\left\{x \in C\left(\left[s, t_{1}\right] ; X\right) \mid\|x(t)-\bar{x}\| \leq 1, s \leq t \leq t_{1}\right\},
$$

where $t_{1}$ would be chosen, and $\|x(t)\| \leq 1+\|\bar{x}\|=\bar{\rho}, s \leq t \leq t_{1} \cdot \mathfrak{B} \subseteq C\left(\left[s, t_{1}\right], X\right)$ is a closed convex set. Define a map $\mathbf{Q}$ on $\mathfrak{B}$ given by

$$
(\mathbf{Q} x)(t)=T(t) \bar{x}+\int_{s}^{t} T(t-\theta) f\left(\theta, x(\theta), \int_{0}^{\theta} g(\theta, s, x(s)) d s\right) d \theta
$$

Under assumptions [H1.1], [H2.1], [H2.2], [H2.4], [H2.5] and Lemma 3.1, one can verify that map $\mathbf{Q}$ is a contraction map on $\mathfrak{B}$ with chosen $t_{1}>0$. This means that system (4.14) has a unique mild solution $x \in C\left(\left[s, t_{1}\right] ; X\right)$ given by

$$
x(t)=T(t) \bar{x}+\int_{s}^{t} T(t-\theta) f\left(\theta, x(\theta), \int_{0}^{\theta} g(\theta, s, x(s)) d s\right) d \theta \quad \text { on }\left[s, t_{1}\right] .
$$


Again, using Lemma 3.1, we can obtain the a priori estimate of the mild solutions for system (4.14) and present the global existence of mild solutions.

Step 2. For $t \in\left(\tau_{k}, \tau_{k+1}\right]$, consider the Cauchy problem

$$
\begin{gathered}
\dot{x}(t)=A x(t)+f\left(t, x, \int_{0}^{t} g(t, s, x) d s\right), \quad t \in\left(\tau_{k}, \tau_{k+1}\right], \\
x\left(\tau_{k}\right)=x_{k} \equiv\left(I+B_{k}\right) x\left(\tau_{k}\right)+c_{k} \in X .
\end{gathered}
$$

By Step 1, Cauchy problem (4.18) also has a unique PC-mild solution

$$
x(t)=T\left(t-\tau_{k}\right) x_{k}+\int_{\tau_{k}}^{t} T(t-\theta) f\left(\theta, x(\theta), \int_{0}^{\theta} g(\theta, s, x(s)) d s\right) d \theta
$$

Step 3. Combining all of the solutions on $\left(\tau_{k}, \tau_{k+1}\right](k=1, \ldots, \delta)$, one can obtain the PC-mild solution of Cauchy problem (4.2) given by

$$
x(t, \bar{x})=S(t, 0) \bar{x}+\int_{0}^{t} S(t, \theta) f\left(\theta, x(\theta), \int_{0}^{\theta} g(\theta, s, x(s)) d s\right) d \theta+\sum_{0 \leq \tau_{k}<t} S\left(t, \tau_{k}^{+}\right) c_{k} .
$$

This completes the proof.

To establish the periodic solutions for system (4.1), we define a Poincaré operator from $X$ to $X$ as follows:

$$
\begin{aligned}
P(\bar{x}) & =x\left(T_{0}, \bar{x}\right) \\
& =S\left(T_{0}, 0\right) \bar{x}+\int_{0}^{T_{0}} S\left(T_{0}, \theta\right) f\left(\theta, x(\theta, \bar{x}), \int_{0}^{\theta} g(\theta, s, x(s, \bar{x})) d s\right) d \theta+\sum_{0 \leq \tau_{k}<T_{0}} S\left(T_{0}, \tau_{k}^{+}\right) c_{k},
\end{aligned}
$$

where $x(\cdot, \bar{x})$ denote the PC-mild solution of Cauchy problem (4.2) corresponding to the initial value $x(0)=\bar{x}$, then we examine whether $P$ has a fixed point.

We first note that a fixed point of $P$ gives rise to a periodic solution.

Lemma 4.5. System (4.1) has a $T_{0}$-periodic PC-mild solution if and only if $P$ has a fixed point.

Proof. Suppose $x(\cdot)=x\left(\cdot+T_{0}\right)$, then $x(0)=x\left(T_{0}\right)=P(x(0))$. This implies that $x(0)$ is a fixed point of $P$. On the other hand, if $P x_{0}=x_{0}, x_{0} \in X$, then for the $P C$-mild solution $x\left(\cdot, x_{0}\right)$ of Cauchy problem (4.2) corresponding to the initial value $x(0)=x_{0}$, we can define 
$y(\cdot)=x\left(\cdot+T_{0}, x_{0}\right)$, then $y(0)=x\left(T_{0}, x_{0}\right)=P x_{0}=x_{0}$. Now, for $t>0$, we can use (2), (3), and (4) of Lemma 2.3 and assumptions [H1.2], [H1.3], [H2.3], [H2.6], and [H2.7] to arrive at

$$
\begin{aligned}
& y(t)=x\left(t+T_{0}, x_{0}\right) \\
& =S\left(t+T_{0}, T_{0}\right) S\left(T_{0}, 0\right) x_{0} \\
& +\int_{0}^{T_{0}} S\left(t+T_{0}, T_{0}\right) S\left(T_{0}, \theta\right) f\left(\theta, x\left(\theta, x_{0}\right), \int_{0}^{\theta} g\left(\theta, s, x\left(s, x_{0}\right)\right) d s\right) d \theta \\
& +\sum_{0 \leq \tau_{k}<T_{0}} S\left(t+T_{0}, T_{0}\right) S\left(T_{0}, \tau_{k}^{+}\right) c_{k} \\
& +\int_{T_{0}}^{t+T_{0}} S\left(t+T_{0}, \theta\right) f\left(\theta, x\left(\theta, x_{0}\right), \int_{0}^{\theta} g\left(\theta, s, x\left(s, x_{0}\right)\right) d s\right) d \theta \\
& +\sum_{T_{0} \leq \tau_{k+\delta}<t+T_{0}} S\left(t+T_{0}, \tau_{k+\delta}^{+}\right) c_{k+\delta} \\
& =S(t, 0)\left\{S\left(T_{0}, 0\right) x_{0}+\int_{0}^{T_{0}} S\left(T_{0}, \theta\right) f\left(\theta, x\left(\theta, x_{0}\right), \int_{0}^{\theta} g\left(\theta, s, x\left(s, x_{0}\right)\right) d s\right) d \theta\right. \\
& \left.+\sum_{0 \leq \tau_{k}<T_{0}} S\left(T_{0}, \tau_{k}^{+}\right) c_{k+\delta}\right\} \\
& +\int_{0}^{t} S\left(t+T_{0}, \theta+T_{0}\right) f\left(\theta+T_{0}, x\left(\theta+T_{0}, x_{0}\right), \int_{0}^{\theta+T_{0}} g\left(\theta+T_{0}, s, x\left(s, x_{0}\right)\right) d s\right) d \theta \\
& +\sum_{T_{0} \leq \tau_{k+\delta}<t+T_{0}} S\left(t+T_{0}, \tau_{k+\delta}^{+}\right) c_{k+\delta} \\
& =S(t, 0) x\left(T_{0}\right)+\int_{0}^{t} S\left(t+T_{0}, \theta+T_{0}\right) f\left(\theta+T_{0}, x\left(\theta+T_{0}, x_{0}\right), \int_{T_{0}}^{\theta+T_{0}} g\left(\theta+T_{0}, s, x\left(s, x_{0}\right)\right) d s\right) d \theta \\
& +\sum_{T_{0} \leq \tau_{k+\delta}<t+T_{0}} S\left(t+T_{0}, \tau_{k+\delta}^{+}\right) c_{k+\delta} \\
& =S(t, 0) x\left(T_{0}\right)+\int_{0}^{t} S(t, \theta) f\left(\theta, x\left(\theta+T_{0}, x_{0}\right), \int_{0}^{\theta} g\left(\theta+T_{0}, s+T_{0}, x\left(s+T_{0}, x_{0}\right)\right) d s\right) d \theta \\
& +\sum_{T_{0} \leq \tau_{k+\delta}<t+T_{0}} S\left(t+T_{0}, \tau_{k+\delta}^{+}\right) c_{k+\delta} \\
& =S(t, 0) y\left(T_{0}\right)+\int_{0}^{t} S(t, \theta) f\left(\theta, y(\theta, y(0)), \int_{0}^{\theta} g(\theta, s, y(s, y(0))) d s\right) d \theta+\sum_{0 \leq \tau_{k}<t} S\left(t, \tau_{k}^{+}\right) c_{k} \\
& =S(t, 0) y(0)+\int_{0}^{t} S(t, \theta) f\left(\theta, y(\theta, y(0)), \int_{0}^{s} g(\theta, s, y(s, y(0))) d s\right) d \theta+\sum_{0 \leq \tau_{k}<t} S\left(t, \tau_{k}^{+}\right) c_{k} .
\end{aligned}
$$


This implies that $y(\cdot, y(0))$ is a PC-mild solution of Cauchy problem (4.2) with initial value $y(0)=x_{0}$. Thus the uniqueness implies that $x\left(\cdot, x_{0}\right)=y(\cdot, y(0))=x\left(\cdot+T_{0}, x_{0}\right)$, so that $x\left(\cdot, x_{0}\right)$ is a $T_{0}$-periodic.

Next, we show that $P$ defined by (4.21) is a continuous and compact operator.

Lemma 4.6. Suppose that $\{T(t), t \geq 0\}$ is a compact semigroup in $X$. Then the operator $P$ is a continuous and compact operator.

Proof. (1) Show that $P$ is a continuous operator on $X$.

Let $\bar{x}, \bar{y} \in \Xi \subset X$, where $\Xi$ is a bounded subset of $X$. Suppose that $x(\cdot, \bar{x})$ and $x(\cdot, \bar{y})$ are the PC-mild solutions of Cauchy problem (4.2) corresponding to the initial values $\bar{x}$ and $\bar{y} \in X$, respectively, given by

$$
\begin{aligned}
& x(t, \bar{x})=S(t, 0) \bar{x}+\int_{0}^{t} S(t, \theta) f\left(\theta, x(\theta, \bar{x}), \int_{0}^{\theta} g(\theta, s, x(s, \bar{x})) d s\right) d \theta+\sum_{0 \leq \tau_{k}<t} S\left(T_{0}, \tau_{k}^{+}\right) c_{k} \\
& x(t, \bar{y})=S(t, 0) \bar{y}+\int_{0}^{t} S(t, \theta) f\left(\theta, x(\theta, \bar{y}), \int_{0}^{\theta} g(\theta, s, x(s, \bar{y})) d s\right) d \theta+\sum_{0 \leq \tau_{k}<t} S\left(T_{0}, \tau_{k}^{+}\right) c_{k} .
\end{aligned}
$$

Thus, we obtain

$$
\begin{aligned}
& \|x(t, \bar{x})\| \\
& \leq M_{T_{0}}\|\bar{x}\|+\left(1+M_{g} T_{0}\right) M_{T_{0}} M_{f} T_{0}+M_{T_{0}} \sum_{0 \leq \tau_{k}<T_{0}}\left\|c_{k}\right\|+M_{T_{0}} M_{f} \int_{0}^{t}\|x(\theta, \bar{x})\| d \theta \\
& \quad+M_{T_{0}} M_{f} M_{g} T_{0} \int_{0}^{\theta}\|x(s, \bar{x})\| d s \\
& \leq a_{0}+M_{T_{0}} M_{f} \int_{0}^{t}\|x(\theta, \bar{x})\| d \theta+M_{T_{0}} M_{f} M_{g} T_{0} \int_{0}^{t}\left\|x_{s, \bar{x}}\right\|_{B} d s, \\
& \|x(t, \bar{y})\| \\
& \leq M_{T_{0}}\|\bar{y}\|+\left(1+M_{g} T_{0}\right) M_{T_{0}} M_{f} T_{0}+M_{T_{0}} \sum_{0 \leq \tau_{k}<T_{0}}\left\|c_{k}\right\|+M_{T_{0}} M_{f} \int_{0}^{t}\|x(\theta, \bar{y})\| d \theta \\
& \quad+M_{T_{0}} M_{f} M_{g} T_{0} \int_{0}^{t}\|x(s, \bar{y})\| d s \\
& \leq \\
& b_{0}+M_{T_{0}} M_{f} \int_{0}^{t}\|x(\theta, \bar{y})\| d \theta+M_{T_{0}} M_{f} M_{g} T_{0} \int_{0}^{t}\left\|x_{s, \bar{y}}\right\|_{B} d s,
\end{aligned}
$$

where $\left\|x_{s, \bar{x}}\right\|_{B}=\sup _{0 \leq \xi \leq s}\|x(\xi, \bar{x})\|$ and $\left\|x_{s, \bar{y}}\right\|_{B}=\sup _{0 \leq \xi \leq s}\|x(\xi, \bar{y})\|$.

By Lemma 3.1, one can verify that there exist constants $M_{1}^{*}$ and $M_{2}^{*}>0$ such that

$$
\|x(t, \bar{x})\| \leq M_{1}^{*}, \quad\|x(t, \bar{y})\| \leq M_{2}^{*}
$$


Let $\rho=\max \left\{M_{1}^{*}, M_{2}^{*}\right\}>0$, then $\|x(\cdot, \bar{x})\|,\|x(\cdot, \bar{y})\| \leq \rho$ which imply that they are locally bounded.

By assumptions [H2.1], [H2.2], [H2.4], [H2.5], and (2) of Lemma 4.3, we obtain

$$
\begin{aligned}
\|x(t, \bar{x})-x(t, \bar{y})\| \leq & \|S(t, 0)\|\|\bar{x}-\bar{y}\| \\
& +\int_{0}^{t}\|S(t, \theta)\| \| f\left(\theta, x(\theta, \bar{x}), \int_{0}^{\theta} g(\theta, s, x(s, \bar{x})) d s\right) \\
& -f\left(\theta, x(\theta, \bar{y}), \int_{0}^{\theta} g(\theta, s, x(s, \bar{y})) d s\right) \| d \theta \\
\leq & M_{T_{0}}\|\bar{x}-\bar{y}\|+M_{T_{0}} L_{f}(\rho) \int_{0}^{t}\|x(\theta, \bar{x})-x(\theta, \bar{y})\| d \theta \\
& +M_{T_{0}} L_{f}(\rho) L_{g}(\rho) T_{0} \int_{0}^{t}\left\|x_{s, \bar{x}}-x_{s, \bar{y}}\right\|_{B} d s .
\end{aligned}
$$

By Lemma 3.1 again, one can verify that there exists constant $M_{3}^{*}>0$ such that

$$
\|x(t, \bar{x})-x(t, \bar{y})\| \leq M_{3}^{*} M_{T_{0}}\|\bar{x}-\bar{y}\| \equiv L\|\bar{x}-\bar{y}\|, \quad \forall t \in\left[0, T_{0}\right]
$$

which implies that

$$
\|P(\bar{x})-P(\bar{y})\|=\left\|x\left(T_{0}, \bar{x}\right)-x\left(T_{0}, \bar{y}\right)\right\| \leq L\|\bar{x}-\bar{y}\| .
$$

Hence, $P$ is a continuous operator on $X$.

(2) Verify that $P$ takes a bounded set into a precompact set in $X$.

Let $\Gamma$ is a bounded subset of $X$. Define $K=P \Gamma=\{P(\bar{x}) \in X \mid \bar{x} \in \Gamma\}$.

For $0<\varepsilon \leq T_{0}$, define

$$
K_{\varepsilon}=P_{\varepsilon} \Gamma=S\left(T_{0}, T_{0}-\varepsilon\right)\left\{x\left(T_{0}-\varepsilon, \bar{x}\right) \mid \bar{x} \in \Gamma\right\} .
$$

Next, we show that $K_{\varepsilon}$ is precompact in $X$. In fact, for $\bar{x} \in \Gamma$ fixed, we have

$$
\begin{aligned}
&\left\|x\left(T_{0}-\varepsilon, \bar{x}\right)\right\| \\
& \leq\left\|S\left(T_{0}-\varepsilon, 0\right) \bar{x}\right\|+\int_{0}^{T_{0}-\varepsilon}\left\|S\left(T_{0}-\varepsilon, \theta\right) f\left(\theta, x(\theta, \bar{x}), \int_{0}^{\theta} g(\theta, s, x(s, \bar{x})) d s\right)\right\| d \theta \\
&+\sum_{0 \leq \tau_{k}<T_{0}-\varepsilon}\left\|S\left(T_{0}-\varepsilon, \tau_{k}^{+}\right) c_{k}\right\|
\end{aligned}
$$


JinRong Wang et al.

$$
\begin{aligned}
& \leq M_{T_{0}}\|\bar{x}\|+M_{T_{0}} M_{f} T_{0}\left(1+M_{g} T_{0}\right)+M_{T_{0}} M_{f} \int_{0}^{T_{0}}\|x(\theta, \bar{x})\| d \theta \\
& \quad+M_{T_{0}} \sum_{0 \leq \tau_{k}<T_{0}}\left\|c_{k}\right\|+M_{T_{0}} M_{f} M_{g} T_{0} \int_{0}^{T_{0}}\left\|x_{s, \bar{x}}\right\|_{B} d s \\
& \leq M_{T_{0}}\|\bar{x}\|+M_{T_{0}} M_{f} T_{0}\left(1+M_{g} T_{0}\right)+\left(1+M_{g} T_{0}\right) M_{T_{0}} M_{f} T_{0} \rho+M_{T_{0}} \sum_{0 \leq \tau_{k}<T_{0}}\left\|c_{k}\right\| .
\end{aligned}
$$

This implies that the set $\left\{x\left(T_{0}-\varepsilon, \bar{x}\right) \mid \bar{x} \in \Gamma\right\}$ is totally bounded.

By virtue of $\{T(t), t \geq 0\}$ which is a compact semigroup and (5) of Lemma 2.3, $S\left(T_{0}, T_{0}-\varepsilon\right)$ is a compact operator. Thus, $K_{\varepsilon}$ is precompact in $X$.

On the other hand, for arbitrary $\bar{x} \in \Gamma$,

$$
\begin{aligned}
P_{\varepsilon}(\bar{x})= & S\left(T_{0}, 0\right) \bar{x}+\int_{0}^{T_{0}-\varepsilon} S\left(T_{0}, \theta\right) f\left(\theta, x(\theta, \bar{x}), \int_{0}^{\theta} g(\theta, s, x(s, \bar{x})) d s\right) d \theta \\
& +\sum_{0 \leq \tau_{k}<T_{0}-\varepsilon} S\left(T_{0}, \tau_{k}^{+}\right) c_{k} .
\end{aligned}
$$

Thus, having this combined with (4.21), we have

$$
\begin{aligned}
& \left\|P_{\varepsilon}(\bar{x})-P(\bar{x})\right\| \\
& \leq \| \int_{0}^{T_{0}-\varepsilon} S\left(T_{0}, \theta\right) f\left(\theta, x(\theta, \bar{x}), \int_{0}^{\theta} g(\theta, s, x(s, \bar{x})) d s\right) d \theta \\
& \quad-\int_{0}^{T_{0}} S\left(T_{0}, \theta\right) f\left(\theta, x(\theta, \bar{x}), \int_{0}^{\theta} g(\theta, s, x(s, \bar{x})) d s\right) d \theta \| \\
& \quad+\left\|\sum_{0 \leq \tau_{k}<T_{0}-\varepsilon} S\left(T_{0}, \tau_{k}^{+}\right) c_{k}-\sum_{0 \leq \tau_{k}<T_{0}} S\left(T_{0}, \tau_{k}^{+}\right) c_{k}\right\| \\
& \leq \int_{T_{0}-\varepsilon}^{T_{0}}\left\|S\left(T_{0}, \theta\right)\right\|\left\|f\left(\theta, x(\theta, \bar{x}), \int_{0}^{\theta} g(\theta, s, x(s, \bar{x})) d s\right)\right\| d \theta+M_{T_{0}} \sum_{T_{0}-\varepsilon \leq \tau_{k}<T_{0}}\left\|c_{k}\right\| \\
& \leq M_{T_{0}} M_{f}\left(1+M_{g} T_{0}\right)(1+\rho) \varepsilon+M_{T_{0}} \sum_{T_{0}-\varepsilon \leq \tau_{k}<T_{0}}\left\|c_{k}\right\| .
\end{aligned}
$$

It is shown that the set $K$ can be approximated to an arbitrary degree of accuracy by a precompact set $K_{\varepsilon}$. Hence $K$ itself is a precompact set in $X$. That is, $P$ takes a bounded set into a precompact set in $X$. As a result, $P$ is a compact operator.

In order to use Leray-Schauder fixed pointed theorem to examine whether the operator $P$ has a fixed point, we have to make assumptions [H2.2] and [H2.5] a little stronger as follows. 
[H2.2'] There exists constant $N_{f}>0$ and $0<\lambda<1$ such that

$$
\|f(t, x, y)\| \leq N_{f}\left(1+\|x\|^{\lambda}+\|y\|^{\lambda}\right) \quad \forall x, y \in X .
$$

[H2.5'] There exists a positive constant $N_{g}>0$ and $0<\lambda<1$ such that

$$
\|g(t, s, x)\| \leq N_{g}\left(1+\|x\|^{\lambda}\right) \quad \forall x \in X
$$

Now, we can give the main results in this paper.

Theorem 4.7. Assumptions [H1], [H2.1], [H2.2'], [H2.3], [H2.4], [H2.5'], [H2.6], and [H2.7] hold. Suppose that $\{T(t), t \geq 0\}$ is a compact semigroup in X. Then system (4.1) has a $T_{0}$-periodic PC-mild solution on $[0,+\infty)$.

Proof. By virtue of $\{T(t), t \geq 0\}$ which is a compact semigroup and (5) of Lemma 2.3, $S\left(T_{0}, 0\right)$ is a compact operator on infinite-dimensional space $X$. Thus, $S\left(T_{0}, 0\right) \neq \alpha I, \alpha \in \mathbb{R}$. Then, there exists $\beta>0$ such that $\left\|\left[\sigma S\left(T_{0}, 0\right)-I\right] \bar{x}\right\| \geq \beta\|\bar{x}\|$ for $\sigma \in[0,1]$. In fact, define $\Pi_{\sigma}=I-\sigma S\left(T_{0}, 0\right)$, $\sigma \in[0,1]$, and $\Pi_{\sigma}:[0,1] \rightarrow £_{b}(X)$ and $h(\sigma)=\left\|\Pi_{\sigma}\right\|:[0,1] \rightarrow \mathbb{R}^{+}$. It is obvious that $h \in$ $C\left([0,1] ; \mathbb{R}^{+}\right)$. Thus, there exist $\sigma_{*} \in[0,1]$ and $\beta>0$ such that

$$
h\left(\sigma_{*}\right)=\min \{h(\sigma) \mid \sigma \in[0,1]\} \geq \beta>0 .
$$

If not, there exists $\bar{\sigma} \in[0,1]$ such that $h(\bar{\sigma})=0$. We can assert that $\bar{\sigma} \neq 0$ unless $h(\bar{\sigma})=1$. Thus, for $\bar{\sigma} \in(0,1]$,

$$
S\left(T_{0}, 0\right)=\frac{1}{\bar{\sigma}} I, \quad \text { where } \frac{1}{\bar{\sigma}} \geq 1,
$$

which is a contradiction with $S\left(T_{0}, 0\right) \neq \alpha I, \alpha \in \mathbb{R}$.

By Theorem 4.4, for fixed $\bar{x} \in X$, Cauchy problem (4.2) corresponding to the initial value $x(0)=\bar{x}$ has the $P C$-mild solution $x(\cdot, \bar{x})$. By Lemma 4.6, the operator $P$ defined by (4.21), is compact.

According to Leray-Schauder fixed point theory, it suffices to show that the set $\{\bar{x} \in$ $X \mid \bar{x}=\sigma P \bar{x}, \sigma \in[0,1]\}$ is a bounded subset of $X$. In fact, let $\bar{x} \in\{\bar{x} \in X \mid \bar{x}=\sigma P \bar{x}, \sigma \in[0,1]\}$, we have

$$
\begin{aligned}
\beta\|\bar{x}\| \leq & \left\|\left[\sigma S\left(T_{0}, 0\right)-I\right] \bar{x}\right\| \\
=\sigma \int_{0}^{T_{0}}\left\|S\left(T_{0}, \theta\right)\right\|\left\|f\left(\theta, x(\theta, \bar{x}), \int_{0}^{\theta} g(\theta, s, x(s, \bar{x})) d s\right)\right\| d \theta & +\sigma \sum_{0 \leq \tau_{k}<T_{0}}\left\|S\left(T_{0}, \tau_{k}^{+}\right)\right\|\left\|c_{k}\right\| .
\end{aligned}
$$


JinRong Wang et al.

By assumptions $\left[\mathrm{H} 2.2^{\prime}\right]$ and $\left[\mathrm{H} 2.5^{\prime}\right]$,

$$
\begin{aligned}
\|\bar{x}\| & \leq \frac{\sigma}{\beta} \int_{0}^{T_{0}}\left\|S\left(T_{0}, \theta\right)\right\|\left\|f\left(\theta, x(\theta, \bar{x}), \int_{0}^{\theta} g(\theta, s, x(s, \bar{x})) d s\right)\right\| d \theta+\frac{\sigma}{\beta} \sum_{0 \leq \tau_{k}<T_{0}}\left\|S\left(T_{0}, \tau_{k}^{+}\right)\right\|\left\|c_{k}\right\| \\
& \leq \frac{\sigma}{\beta} M_{T_{0}}\left(\left(N_{f}+N_{g} T_{0}\right) T_{0}+N_{f} \int_{0}^{T_{0}}\|x(\theta, \bar{x})\|^{\lambda} d \theta+N_{f} N_{g} T_{0} \int_{0}^{T_{0}}\left\|x_{s, \bar{x}}\right\|_{B}^{\lambda} d \theta+\sum_{0 \leq \tau_{k}<T_{0}}\left\|c_{k}\right\|\right) .
\end{aligned}
$$

For $t \in\left[0, T_{0}\right]$, we obtain

$$
\begin{aligned}
& \|x(t, \bar{x})\| \\
& \leq M_{T_{0}}\|\bar{x}\|+M_{T_{0}}\left(\left(N_{f}+N_{g} T_{0}\right) T_{0}+N_{f} \int_{0}^{t}\|x(\theta, \bar{x})\|^{\lambda} d \theta+N_{f} N_{g} T_{0} \int_{0}^{t}\left\|x_{s, \bar{x}}\right\|_{B}^{\lambda} d \theta+\sum_{0 \leq \tau_{k}<t}\left\|c_{k}\right\|\right) \\
& \leq \frac{\sigma}{\beta} M_{T_{0}}^{2}\left(\left(N_{f}+N_{g} T_{0}\right) T_{0}+N_{f} \int_{0}^{T_{0}}\|x(\theta, \bar{x})\|^{\lambda} d \theta+N_{f} N_{g} T_{0} \int_{0}^{T_{0}}\left\|x_{s, \bar{x}}\right\|_{B}^{\lambda} d \theta+\sum_{0 \leq \tau_{k}<T_{0}}\left\|c_{k}\right\|\right) \\
& +M_{T_{0}}\left(\left(N_{f}+N_{g} T_{0}\right) T_{0}+N_{f} \int_{0}^{t}\|x(\theta, \bar{x})\|^{\lambda} d \theta+N_{f} N_{g} T_{0} \int_{0}^{t}\left\|x_{s, \bar{x}}\right\|_{B}^{\lambda} d \theta+\sum_{0 \leq \tau_{k}<t}\left\|c_{k}\right\|\right) \\
& \leq\left(\frac{\sigma}{\beta} M_{T_{0}}+1\right) M_{T_{0}}\left(\left(N_{f}+N_{g} T_{0}\right) T_{0}+\sum_{0 \leq \tau_{k}<T_{0}}\left\|c_{k}\right\|\right) \\
& \quad+M_{T_{0}} N_{f} \int_{0}^{t}\|x(\theta, \bar{x})\|^{\lambda} d \theta+\frac{\sigma}{\beta} M_{T_{0}}^{2} N_{f} \int_{0}^{T_{0}}\|x(\theta, \bar{x})\|^{\lambda} d \theta+M_{T_{0}} N_{f} N_{g} T_{0} \int_{0}^{t}\left\|x_{s, \bar{x}}\right\|_{B}^{\lambda} d \theta \\
& \quad+\frac{\sigma}{\beta} M_{T_{0}}^{2} N_{f} N_{g} T_{0} \int_{0}^{T_{0}}\left\|x_{s, \bar{x}}\right\|_{B}^{\lambda} d \theta .
\end{aligned}
$$

By Lemma 3.2, there exists $M^{*}>0$ such that

$$
\|x(t, \bar{x})\| \leq M^{*} \quad \text { for } t \in\left[0, T_{0}\right]
$$

This implies that $\|x(0, \bar{x})\|=\|\bar{x}\| \leq M^{*}$ for all $\bar{x} \in\{\bar{x} \in X \mid \bar{x}=\sigma P \bar{x}, \sigma \in[0,1]\}$.

Thus, by Leray-Schauder fixed pointed theory, there exists $x_{0} \in X$ such that $P x_{0}=$ $x_{0}$. By Lemma 4.5, we know that the $P C$-mild solution $x\left(\cdot, x_{0}\right)$ of Cauchy problem (4.2) corresponding to the initial value $x(0)=x_{0}$ is just $T_{0}$-periodic. Therefore $x\left(\cdot, x_{0}\right)$ is a $T_{0^{-}}$ periodic PC-mild solution of system (4.1). 


\section{Application}

In this section, an example is given to illustrate our theory. Consider the following problem:

$$
\begin{gathered}
\frac{\partial}{\partial t} x(t, y)=\Delta x(t, y)+x^{2 / 3}(t, y)+\int_{0}^{t} \psi(s)(1+\sin (t-s)) \sqrt{3 x^{2 / 3}(s, y)+2} d s, \\
y \in \Omega, \quad t, s \in(0,2 \pi] \backslash\left\{\frac{1}{2} \pi, \pi, \frac{3}{2} \pi\right\}, \\
\Delta x\left(\tau_{i}, y\right)=x\left(\tau_{i}^{+}, y\right)-x\left(\tau_{i}^{-}, y\right)= \begin{cases}0.05 I x\left(\tau_{i}, y\right), & i=1, \\
-0.05 I x\left(\tau_{i}, y\right), & i=2, y \in \Omega, \tau_{i}=\frac{i}{2} \pi, i=1,2,3, \\
0.05 I x\left(\tau_{i}, y\right), & i=3,\end{cases} \\
\left.x(t, y)\right|_{y \in \partial \Omega}=0, \quad t>0, \\
x(0, y)=x(2 \pi, y),
\end{gathered}
$$

where $\Omega \subset \mathbb{R}^{3}$ is bounded domain and $\partial \Omega \in C^{3}$.

Define $X=L_{2}(\Omega), D(A)=H^{2}(\Omega) \cap H_{0}^{1}(\Omega)$, and $A x=-\left(\partial^{2} x / \partial y_{1}^{2}+\partial^{2} x / \partial y_{2}^{2}+\right.$ $\left.\partial^{2} x / \partial y_{3}^{2}\right)$ for $x \in D(A)$. Then, $A$ generates a compact semigroup $\{T(t), t \geq 0\}$. Define $x(\cdot)(y)=x(\cdot, y), \sin (\cdot)(y)=\sin (\cdot, y), f\left(\cdot, x(\cdot), \int_{0} g(\cdot, s, x) d s\right)(y)=x^{2 / 3}(\cdot)(y)+\int_{0}^{t} \psi(s)(1+$ $\sin (\cdot-s)) \sqrt{3 x^{2 / 3}(\cdot)+2} d s(y)$, where $\psi(\cdot+2 \pi)=\psi(\cdot) \in L_{\text {loc }}^{1}([0,+\infty) ; X), \int_{0}^{2 \pi} \psi(s)(1+\sin (t-$ s)) $\sqrt{3 x^{2 / 3}(t)+2} d s=0$, and

$$
B_{i}= \begin{cases}0.05 I, & i=1, \\ -0.05 I, & i=2, \\ 0.05 I, & i=3 .\end{cases}
$$

Thus problem (5.1) can be rewritten as

$$
\begin{gathered}
\dot{x}(t)=A x(t)+f\left(t, x, \int_{0}^{t} g(t, s, x) d s\right), \quad t \in(0,2 \pi] \backslash\left\{\frac{1}{2} \pi, \pi, \frac{3}{2} \pi\right\}, \\
\Delta x\left(\frac{i}{2} \pi\right)=B_{i} x\left(\frac{i}{2} \pi\right), \quad i=1,2,3, \\
x(0)=x(2 \pi) .
\end{gathered}
$$


It satisfies all the assumptions given in Theorem 4.7, our results can be used to problem (5.1). That is, problem (5.1) has a $2 \pi$-periodic $P C$-mild solution $x_{2 \pi}(\cdot, y) \in P C_{2 \pi}([0+$ $\infty) ; L_{2}(\Omega)$ ), where

$$
P C_{2 \pi}\left([0,+\infty) ; L_{2}(\Omega)\right) \equiv\left\{x \in P C\left([0,+\infty) ; L_{2}(\Omega)\right) \mid x(t)=x(t+2 \pi), t \geq 0\right\} .
$$

\section{Acknowledgments}

This work is supported by National Natural Science foundation of China (no. 10661044) and Natural Science Foundation of Guizhou Province Education Department (no. 2007008). This work is also partially supported by undergraduate carve-out project of Department of Guiyang City Science and Technology.

\section{References}

[1] D. D. Bainov and P. S. Simeonov, Impulsive Differential Equations: Periodic Solutions and Applications, vol. 66 of Pitman Monographs and Surveys in Pure and Applied Mathematics, Longman Scientific \& Technical, Harlow, UK, 1993.

[2] V. Lakshmikantham, D. D. Bainov, and P. S. Simeonov, Theory of Impulsive Differential Equations, vol. 6 of Series in Modern Applied Mathematics, World Scientific, Teaneck, NJ, USA, 1989.

[3] X. Liu, "Impulsive stabilization and applications to population growth models," The Rocky Mountain Journal of Mathematics, vol. 25, no. 1, pp. 381-395, 1995.

[4] T. Yang, Impulsive Control Theory, vol. 272 of Lecture Notes in Control and Information Sciences, Springer, Berlin, Germany, 2001.

[5] N. U. Ahmed, "Optimal impulse control for impulsive systems in Banach spaces," International Journal of Differential Equations and Applications, vol. 1, no. 1, pp. 37-52, 2000.

[6] N. U. Ahmed, "Some remarks on the dynamics of impulsive systems in Banach spaces," Dynamics of Continuous, Discrete and Impulsive Systems. Series A, vol. 8, no. 2, pp. 261-274, 2001.

[7] N. U. Ahmed, K. L. Teo, and S. H. Hou, "Nonlinear impulsive systems on infinite dimensional spaces," Nonlinear Analysis: Theory, Methods \& Applications, vol. 54, no. 5, pp. 907-925, 2003.

[8] N. U. Ahmed, "Existence of optimal controls for a general class of impulsive systems on Banach spaces," SIAM Journal on Control and Optimization, vol. 42, no. 2, pp. 669-685, 2003.

[9] M. Benchohra, J. Henderson, S. K. Ntouyas, and A. Ouahab, "Multiple solutions for impulsive semilinear functional and neutral functional differential equations in Hilbert space," Journal of Inequalities and Applications, vol. 2005, no. 2, pp. 189-205, 2005.

[10] N.-E. Tatar, "An impulsive nonlinear singular version of the Gronwall-Bihari inequality," Journal of Inequalities and Applications, vol. 2006, Article ID 84561, 12 pages, 2006.

[11] D. J. Guo and X. Z. Liu, "Extremal solutions of nonlinear impulsive integrodifferential equations in Banach spaces," Journal of Mathematical Analysis and Applications, vol. 177, no. 2, pp. 538-552, 1993.

[12] J. H. Liu, "Bounded and periodic solutions of differential equations in Banach space," Applied Mathematics and Computation, vol. 65, no. 1-3, pp. 141-150, 1994.

[13] J.-Y. Park, Y.-C. Kwun, and J.-M. Jeong, "Existence of periodic solutions for delay evolution integrodifferential equations," Mathematical and Computer Modelling, vol. 40, no. 5-6, pp. 597-603, 2004.

[14] K. Liu and G. Yang, "Strict stability criteria for impulsive functional differential systems," Journal of Inequalities and Applications, vol. 2008, Article ID 243863, 8 pages, 2008.

[15] W. Wei, X. Xiang, and Y. Peng, "Nonlinear impulsive integro-differential equations of mixed type and optimal controls," Optimization, vol. 55, no. 1-2, pp. 141-156, 2006.

[16] X. Xiang and W. Wei, "Mild solution for a class of nonlinear impulsive evolution inclusions on Banach space," Southeast Asian Bulletin of Mathematics, vol. 30, no. 2, pp. 367-376, 2006.

[17] X. Xiang, W. Wei, and Y. Jiang, "Strongly nonlinear impulsive system and necessary conditions of optimality," Dynamics of Continuous, Discrete and Impulsive Systems. Series A, vol. 12, no. 6, pp. 811$824,2005$.

[18] X. Yu, X. Xiang, and W. Wei, "Solution bundle for a class of impulsive differential inclusions on Banach spaces," Journal of Mathematical Analysis and Applications, vol. 327, no. 1, pp. 220-232, 2007. 
[19] Y. Peng, X. Xiang, and W. Wei, "Nonlinear impulsive integro-differential equations of mixed type with time-varying generating operators and optimal controls," Dynamic Systems and Applications, vol. 16, no. 3, pp. 481-496, 2007.

[20] Y. Peng and X. Xiang, "Second order nonlinear impulsive time-variant systems with unbounded perturbation and optimal controls," Journal of Industrial and Management Optimization, vol. 4, no. 1, pp. 17-32, 2008.

[21] H. Amann, "Periodic solutions of semilinear parabolic equations," in Nonlinear Analysis: A Collection of Papers in Honor of Erich H. Rothe, pp. 1-29, Academic Press, New York, NY, USA, 1978.

[22] P. Sattayatham, S. Tangmanee, and W. Wei, "On periodic solutions of nonlinear evolution equations in Banach spaces," Journal of Mathematical Analysis and Applications, vol. 276, no. 1, pp. 98-108, 2002.

[23] X. Xiang and N. U. Ahmed, "Existence of periodic solutions of semilinear evolution equations with time lags," Nonlinear Analysis: Theory, Methods \& Applications, vol. 18, no. 11, pp. 1063-1070, 1992.

[24] J. Wang, "Linear impulsive periodic system on Banach space," in Proceedings of the 4th International Conference on Impulsive and Hybrid Dynamical Systems (ICIDSA '07), vol. 5, pp. 20-25, Nanning, China, July 2007.

[25] J. Wang, X. Xiang, and W. Wei, "Linear impulsive periodic system with time-varying generating operators on Banach space," Advances in Difference Equations, vol. 2007, Article ID 26196, 16 pages, 2007.

[26] J. Wang, X. Xiang, W. Wei, and Q. Chen, “Existence and global asymptotical stability of periodic solution for the $T$-periodic logistic system with time-varying generating operators and $T_{0}$-periodic impulsive perturbations on Banach spaces," Discrete Dynamics in Nature and Society, vol. 2008, Article ID 524945, 16 pages, 2008.

[27] J. Wang, X. Xiang, W. Wei, and Q. Chen, "Bounded and periodic solutions of semilinear impulsive periodic system on Banach spaces," Fixed Point Theory and Applications, vol. 2008, Article ID 401947, 15 pages, 2008.

[28] J. Wang, X. Xiang, and W. Wei, "Existence and stability of periodic solution for periodic logistic system with periodic impulsive perturbations," in Proceedings of the 4th International Conference on Natural Computation, vol. 1, pp. 197-201, 2008.

[29] N. U. Ahmed, Semigroup Theory with Applications to Systems and Control, vol. 246 of Pitman Research Notes in Mathematics Series, Longman Scientific \& Technical, Harlow, UK, 1991. 